AperTO - Archivio Istituzionale Open Access dell'Università di Torino

\title{
A molecular exploration of human DNA/RNA co-extracted from the palmar surface of the hands and fingers
}

\section{This is the author's manuscript}

Original Citation:

Availability:

This version is available http://hdl.handle.net/2318/1557364

since 2016-11-07T16:57:10Z

Published version:

DOI:10.1016/j.fsigen.2016.01.012

Terms of use:

Open Access

Anyone can freely access the full text of works made available as "Open Access". Works made available under a Creative Commons license can be used according to the terms and conditions of said license. Use of all other works requires consent of the right holder (author or publisher) if not exempted from copyright protection by the applicable law. 


\section{UNIVERSITÀ DEGLI STUDI DI TORINO}

This Accepted Author Manuscript (AAM) is copyrighted and published by Elsevier. It is posted here by agreement between Elsevier and the University of Turin. Changes resulting from the publishing process - such as editing, corrections, structural formatting, and other quality control mechanisms may not be reflected in this version of the text. The definitive version of the text was subsequently published in [Forensic Science International: Genetics, 22, 2016 Jan 21, doi: 10.1016/j.fsigen.2016.01.012.].

You may download, copy and otherwise use the AAM for non-commercial purposes provided that your license is limited by the following restrictions:

(1) You may use this AAM for non-commercial purposes only under the terms of the CC-BY-NC-ND license.

(2) The integrity of the work and identification of the author, copyright owner, and publisher must be preserved in any copy.

(3) You must attribute this AAM in the following format: Creative Commons BY-NC-ND license (http://creativecommons.org/licenses/by-nc-nd/4.0/deed.en), [doi: 10.1016/j.fsigen.2016.01.012.] 
A molecular exploration of human DNA/RNA co-extracted from the palmar surface of the hands and fingers

Lacerenza $D^{a}$, Aneli $S^{a, b}$, Omedei $M^{a, c}$, Gino $S^{a}$, Pasino $S^{a}$, Berchialla $P^{d}$, and Robino $C^{a^{*}}$

${ }^{a}$ Department of Public Health Sciences and Pediatrics, University of Turin, Turin, Italy

${ }^{\mathrm{b}}$ Human Genetics Foundation, HuGeF, Turin, Italy

'Department of Public Health, University of Rome “Tor Vergata”, Rome, Italy

${ }^{\mathrm{d}}$ Department of Clinical and Biological Sciences, University of Turin, Turin, Italy

* Corresponding author: Department of Public Health Sciences and Pediatrics, University of Turin, Corso Galileo Galilei 22, 10126 Turin, Italy

e-mail: carlo.robino@unito.it (C. Robino)

\section{Abstract}

"Touch DNA" refers to the DNA that is left behind when a person touches or comes into contact with an item. However, the source of touch DNA is still debated and the large variability in DNA yield from casework samples suggests that, besides skin, various body fluids can be transferred through contact. Another important issue concerning touch DNA is the possible occurrence of secondary transfer, but the data published in the literature in relation to the background levels of foreign DNA present on the hand surfaces of the general population are very limited.

As the present study aimed at better understanding the nature and characteristics of touch DNA, samples were collected from the palmar surface of the hands and fingers ("PHF" samples) of 30 male and 30 female donors by tape-lifting/swabbing and subjected to DNA/RNA co-extraction. Multiplex mRNA profiling showed that cellular material different from skin could be observed in $15 \%$ of the PHF samples. The total amount of DNA recovered from these samples (median 5.1 
ng) was significantly higher than that obtained from samples containing skin cells only (median $1.6 \mathrm{ng})$.

The integrity of the DNA isolated from the donors' hands and fingers as well as the prevalence of DNA mixtures were evaluated by STR typing and compared with reference STR profiles from buccal swabs. DNA integrity appeared significantly higher in the male rather than in the female subsample, as the average percentage of the donors' alleles effectively detected in PHF profiles was $75.1 \%$ and $60.1 \%$ respectively. The prevalence of mixtures with a foreign DNA contribution $\geq 20 \%$ was $19.2 \%$ (30.0\% in the female PHF samples and $8.3 \%$ in the male PHF samples).

The obtained results support the hypothesis that transfer of cellular material different from skin may underlie the occasional recovery of quality STR profiles from handled items. These results also suggest that gender may represent an important factor influencing the propensity of individuals to carry and transfer DNA through hand contact, possibly because of the differences in personal and hygiene habits between males and females.

\section{Keywords}

Touch DNA, hand, DNA/RNA co-extraction, mRNA profiling, DNA mixture

\section{Introduction}

The increasing sensitivity of multiplex PCR assays for the simultaneous amplification of Short Tandem Repeat (STR) loci enables forensic investigators to recover DNA profiles even from tiny stains and highly degraded samples, including cellular residues left behind when a person touches or comes into contact with an item (i.e., the so-called "touch DNA"). The first report on the possibility to generate genetic profiles from swabs taken from handled objects dates back to 1997 
[1]. Since that pioneering study, however, considerable uncertainty has remained about several aspects of touch DNA, i.e., its biological sources, composition, transfer dynamics and ability to withstand different environmental conditions [2,3].

It is often assumed that touch DNA derives from a limited number of shed nucleated cells and of stripped nuclei dispersed among mostly anucleate corneocytes, which can be found on the surface of the outermost layer of the epidermis [4,5]. It has recently been suggested that DNA recovered from touched surfaces may not entirely originate from epidermal cells, but that it could also consist of cell-free DNA from extracellular secretions like sweat and sebum [6,7]. Moreover, since skin is by nature constantly exposed to the external environment, it is expected to carry on its surface body fluids that do not derive from the epidermis and its annexes, and to consequently transfer them to objects through handling. For instance, saliva seems to be ubiquitous in the human environment, as it is diffused via droplets originating from the mouth [8]; in fact, it was demonstrated that a static speaking individual can rapidly deposit in the surrounding area a sufficient amount of DNA for a positive identification [9]. Hence, wearing protective masks at the crime scene is mandatory to prevent contamination [10]. Because of the relatively high cellular content of human saliva, DNA yields from hand surfaces subjected to primary transfer of the same fluid are significantly higher than those obtained from freshly washed, bare palms [11]. It can therefore be hypothesized that, rather than the individual propensity to transfer DNA (i.e., the so-called "shedder status" [12]), the presence on human hands of body fluids different from skin may be the reason for the high quantities of human DNA that are sporadically detected on handled objects (both experimental and casework samples) [1,13-16].

Another important issue concerning touch DNA is the possible occurrence of secondary transfer, i.e., the indirect transfer of the donor's DNA to a surface via an intermediary who, in turn, has either come into direct contact with the donor or held an object previously touched by the donor 
[1]. Therefore, to better understand the possible extent of secondary transfer events, an assessment of the background levels of foreign DNA present on the hand surfaces of the general population appears to be crucial. Nevertheless, previous studies have almost exclusively focused on the prevalence of DNA mixtures under fingernails [17-19], largely ignoring the palm and finger surfaces of the hand, which are the anatomical parts more frequently involved in object handling and in the transfer of touch DNA.

Recently, several robust protocols for DNA/RNA co-extraction from stains have been described and validated for forensic purposes [20], thus enabling the single pipeline analysis of both STRs and mRNA profiles to identify body fluids. In particular, the availability of skin-specific mRNA markers $[21,22]$ now offers the possibility of better exploring the tissue origin of touch DNA. Hence, the aim of the present study was to investigate the characteristics of the human biological material present on the palmar surface of the hands and fingers of individuals from the general population through combined DNA/mRNA profiling. The mRNA profiling results were then put in relation with other factors that are relevant for the interpretation of touch DNA dynamics such as DNA quantity and integrity, prevalence of DNA mixtures and percentage contribution of foreign DNA to mixtures.

\section{Materials and Methods}

\subsection{Sample collection}

After the Ethical Committee of the University of Turin granted approval for the study, consenting participants were recruited among university students and laboratory personnel. Although donors were informed of the nature and purpose of the experiments, they were not notified in advance of the exact date and time of sampling so as to prevent changes in their activities and social interactions in the period immediately preceding sample collection. 
Samples $(n=120)$ were collected from the palmar surface of the hands and fingers of 60 different donors (30 males and 30 females) and are referred to from now on as "PHF" samples. All donors were sampled once, providing separate PHF samples from each hand. Three different sampling methods were employed: dry sterile cotton swab (10 males and 10 females); sterile cotton swab moistened with RNase-free water (10 males and 10 females); tape lift by means of "stubs" that are currently used for gunshot residue collection (10 males and 10 females). A buccal swab was also collected from each individual to establish reference STR profiles. Participation in the study was anonymous and the only information recorded at the time of sampling included age, gender and hand dominance. Donors were also asked to complete a questionnaire (Supplementary material, Table S1) to record their latest activities, social interactions, personal and hygiene habits, etc.

\subsection{DNA/RNA co-extraction}

After collection, swabs/stubs were air-dried and stored at room temperature for 6-24 h before being submitted to nucleic acid isolation. Total DNA and RNA were extracted using the AllPrep DNA/RNA Micro Kit (Qiagen, Hilden, Germany), according to the manufacturer's instructions. For the disruption and homogenization of the starting material, swabs/stubs were placed in a spin basket (Investigator Lyse\&Spin Basket Kit, Qiagen) with $345 \mu 1$ RLT buffer, $5 \mu$ l Carrier RNA (4 $\mathrm{ng} / \mu \mathrm{l})$ and $13.8 \mu \mathrm{DTT}(1 \mathrm{M})$, and incubated $3 \mathrm{~h}$ at $56^{\circ} \mathrm{C}$. Lysate collection was then performed by centrifugation at maximum speed. DNA and RNA were finally eluted in $50 \mu 1$ of buffer EB and $13 \mu 1$ of RNase-free water respectively.

Genomic DNA was isolated from buccal swabs using the ChargeSwitch gDNA Normalized Buccal Cell Kit (Invitrogen, Carlsbad, CA, USA) and the KingFisher mL Magnetic separator (Thermo Fisher Scientific, Vantaa, Finland). 


\section{$2.3 \quad$ mRNA profiling}

RNA extracts were treated with DNase (TURBO DNA free, Ambion, Carlsbad, CA, USA) and cDNA was synthesized using the RETROscript Kit with Random Decamers (Ambion), according to the manufacturer's instructions.

Sixteen tissue markers (HBB, CD93, AMICA1: blood; KRT4, SPRR2A, KRT13: mucosa; HTN3, STATH: saliva; SEMG1, PRM1: semen; HBD1, MUC4: vaginal mucosa; MMP7, MMP11: menstrual secretions; CDSN, LOR: skin) and three housekeeping genes (GAPDH, 18S-rRNA and ACTB) were simultaneously amplified by multiplex end-point PCR with a range of different cDNA inputs $(7,3.5,1 \mu \mathrm{l})$ and detected by capillary electrophoresis on the ABI Prism 310 Genetic Analyzer (Life Technologies, Foster City, CA, USA), as described by Lindenbergh et al. [23]

\subsection{Body fluid classification}

On the basis of the mRNA profiling results, PHF samples were assigned to three broad tissue type categories: "skin", "other than skin", "indeterminate". Classification was based on the scoring guidelines introduced by Lindenbergh et al. in a previous study [24]. In brief, informative profiles (i.e., those showing the highest number of expressed housekeeping genes and no saturated peaks caused by overexpression of single tissue markers) were selected among the mRNA profiling results obtained with different cDNA inputs. Further RT-PCR reactions were then performed using the specific cDNA inputs that had previously generated an informative profile so as to obtain at least three informative mRNA profiling replicates for each sample. Moreover, for samples where the menstrual secretion and/or the vaginal mucosa markers were expressed, amplification of HBD1 was also performed in a singleplex reaction [23]. PHF samples were 
classified as containing "skin" if peaks at skin-specific marker positions (CDSN, LOR) were "observed", i.e., were present in at least $50 \%$ of the replicates. Since co-expression of general mucosa (KRT4, SPRR2A, KRT13) and skin markers is frequently detected [23], samples where the same markers were observed but no expression of other tissue markers was found, were also classified as skin. PHF samples were classified as "other than skin" when markers for body fluids different from skin were observed. Finally, samples showing either no mRNA signals or the expression of housekeeping genes only were classified as "indeterminate". This last category also included samples where general mucosa markers were observed and skin as well as other body fluid markers were absent.

\subsection{DNA quantitation and typing}

Human genomic DNA isolated from palmar surfaces was quantified with the Plexor® HY System (Promega, Madison, IL, USA) and the CFX96 Touch Real-Time PCR Detection System (BioRad, Hercules, CA, USA). STR typing was performed using the Investigator ESSplex SE Plus Kit (Qiagen). A recommended amount of $0.5 \mathrm{ng}$ of template DNA was included in the PCR whenever possible. For PHF samples showing suboptimal DNA concentrations, the maximum volume of input DNA allowed per reaction $(15 \mu \mathrm{l})$ was employed. Amplifications were then carried out using a standard 30-cycle PCR protocol, according to the manufacturer's instructions. Allele detection was performed by capillary electrophoresis (ABI Prism 310 Genetic Analyzer, Life Technologies); the results were processed using the GeneMapper software (Life Technologies) and the provided template file, which includes locus-specific stutter filters and a set analytical threshold of 50 rfu (Analysis_HID_310_50_rfu, Qiagen).

\subsection{Evaluation of DNA “integrity”}


The integrity of the DNA isolated from the donors' hands and fingers was evaluated comparing the obtained STR profiles with the aforementioned reference profiles derived from buccal samples. In particular, integrity was calculated as follows: $\mathrm{N}_{\mathrm{oe}} / \mathrm{N}_{\mathrm{e}} \mathrm{x} 100$, where $\mathrm{N}_{\mathrm{oe}}$ is the number of alleles expected from each donor's reference profile and effectively observed in the donor's palmar surface sample, and $\mathrm{N}_{\mathrm{e}}$ is the whole number of expected alleles. The amelogenin locus included in the ESSplex SE Plus Panel was not considered for calculations because of the possible gender bias due to the fact that male donors have two ( $\mathrm{Y}$ - and $\mathrm{X}$-specific) peaks that may be susceptible to drop-out, in contrast with female donors who only have the X-specific peak.

\subsection{Identification of DNA mixtures and estimation of foreign DNA contribution}

According to Cook et al. [17], STR profiles of PHF samples were classified as mixtures when $\geq 4$ unexpected allele peaks, identified on the basis of the donor's reference profile, could be located in more than one of the STR loci. PHF samples with less than 4 foreign alleles were considered as "low-level" mixtures. Since the detection of mixtures is impossible by simply considering the presence of an unexpected allele at the amelogenin locus in males (who already show all the possible alleles in their reference genotype), this marker was excluded from calculations.

The percentage contribution of foreign DNA to mixtures was calculated for each PHF sample profile as follows: $H_{u} / H_{0} \times 100$, where $H_{u}$ is the total peak height of unexpected alleles with respect to the donor's reference profile, and $\mathrm{H}_{\mathrm{o}}$ is the total peak height of all the alleles observed in the donor's PHF sample.

\subsection{Statistical analysis}

The Chi-square test was employed to compare frequencies. As for distributions, normality was first verified by means of both a graphical evaluation and the Shapiro-Wilk test; the Wilcoxon 
Rank Sum test and the Kruskal-Wallis test were used to identify statistically significant differences between non-normally distributed variables. Appropriate adjustments were applied to the Chi-square [25] and the Wilcoxon test [26] so as to take into account the clustered nature of the data (i.e., pairs of hands). The Pearson's correlation coefficient was computed with the aim of testing the association between paired samples.

To assess which of the considered factors (i.e., sampling method, gender, hand dominance, tissue type category) had mostly influenced DNA yield, integrity and mixtures in PHF samples, either a multivariate linear regression or a quantile regression analysis was performed, depending on the distributional assumptions. The Huber-White sandwich estimator was used in the multivariate linear regression analysis so as to take into account the clustered nature of data and to obtain robust standard errors. For the same reason, a linear quantile mixed effect framework was adopted in the quantile regression analysis.

A latent class analysis (LCA) was performed to identify the profiles associated with different amounts of recovered DNA and with different percentages of foreign DNA contribution to mixtures. Two latent class models were fitted, one for each concomitant variable. The LCA required the specification of the number of clusters (latent classes). A series of models was fitted for two to five clusters. The best model was selected on the basis of the lowest value of the Bayesian Information Criterion. The estimated class-conditional response probabilities and a matrix containing each observation's posterior class membership probabilities were also retrieved. Analyses were conducted using R version 3.2 [27].

\section{Results}

\subsection{Sampling conditions and age distribution of the donors}


On sampling days, the average temperature was $17^{\circ} \mathrm{C}$ (range $11-22^{\circ} \mathrm{C}$ ), with an average relative humidity of $48.4 \%$ (range $32-62 \%$ ).

The median age of donors was 23 years (range 18-66 years). The median age in the female subsamples was 23 years (range 18-63 years), whereas in the male subsamples was 28 years (range 18-66 years).

\section{2 mRNA profiling and tissue classification}

According to the mRNA profiling and the above-mentioned scoring guidelines, $55 \%$ of the PHF samples were classified as containing only skin. Markers specific for body fluids different from skin were observed in $15 \%$ of the PHF samples. In all but one of these samples (17 out of 18$)$ skin markers were also observed. Within the indeterminate category (28\%), the proportion of samples with observed general mucosa markers (i.e., samples that could not fall within the skin or the other than skin categories due to the absence of more specific tissue markers) was negligible (1 out of 34 samples).

Tissue composition of other than skin samples is shown in Supplementary Figure S1. Blood and saliva were the most common body fluids and were both detected in $33 \%$ of the PHF samples.

Table 1 shows the frequency of tissue types subdivided by sampling method, gender, and hand dominance. The only significant difference was related to the sampling methods, as the wet swab method generated a significantly higher percentage of indeterminate results than the dry swab and the tape lift ones (Chi-square adjusted for clustered data, $\mathrm{p}=0.022$ ). An interesting feature related to gender was that, although the occurrence of other than skin material was not significantly higher in males than in females, the first ones showed a wider variety of tissue types on their palmar surfaces compared to the second ones. Female other than skin samples $(n=5)$ always included no more than one body fluid type different from skin, with blood representing the most 
common tissue (found in two PHF samples), followed by saliva, vaginal mucosa and menstrual secretion (one PHF sample each). Of the male PHF samples classified as other than skin $(n=13)$, five (38.5\%) consisted in mixtures of $\geq 2$ tissue types different from skin, and four (30.8\%) included body fluids typical of the other sex (i.e., vaginal mucosa, either alone or in combination with other body fluids).

\subsection{DNA yield from PHF samples}

The total amount of DNA recovered from PHF samples ranged between 0 and $585 \mathrm{ng}$ (median 1.6 ng, IQR: Q1 0.5 - Q3 5). The box-and-whisker plots (see Figure 1) show the distribution of DNA yields from samples subdivided by sampling method, gender, hand dominance, and tissue type category. Significant differences were observed in relation to gender, with male donors (median $3.6 \mathrm{ng}$ ) showing higher DNA yields than female donors (median $1.0 \mathrm{ng}$ ) (Wilcoxon test corrected for clustered data, adjusted for multiplicity, $\mathrm{p}<0.001$ ). As for tissue type, DNA recovery was significantly higher in other than skin samples (median $5.1 \mathrm{ng}$ ) than in skin samples (median 1.6 ng) (Wilcoxon test corrected for clustered data, adjusted for multiplicity, $\mathrm{p}=0.010$ ). DNA yields from both other than skin and skin samples were significantly higher compared to the yields from the indeterminate samples (median $0.8 \mathrm{ng}$ ) (Wilcoxon test corrected for clustered data, adjusted for multiplicity, $p=0.002$ and $p=0.010$ respectively). Conversely, no significant differences were found in relation to sampling method and hand dominance.

With the aim of testing which of the considered factors mostly influenced DNA yield, a multivariate linear regression was performed. Gender still remained significant $(p=0.008)$, along with tissue type (indeterminate vs. other than skin, $p=0.001$; skin vs. other than skin, $p=0.018$ ) and wet swab ( $\mathrm{p}=0.038)$, which was in turn associated with higher DNA yields if compared to the other sampling methods. 


\subsection{DNA integrity in PHF samples}

The proportion of PHF samples generating high-integrity profiles (i.e., showing $\geq 90 \%$ of the expected STR alleles) was 56\%. A positive correlation between DNA yield and integrity was observed (Pearson's coefficient 0.540, $\mathrm{p}<0.001$ ). Integrity percentages for samples subdivided by sampling method, gender, hand dominance and tissue type category are shown in Table 2 . Significant differences always involved pairs of categories that had already shown to differ with regard to DNA yield: males (average integrity $75.1 \% \pm 5.2 \% \mathrm{SE}$ ) vs. females (average integrity $60.1 \% \pm 5.2 \% \mathrm{SE}$ ) (Wilcoxon test corrected for clustered data, $\mathrm{p}=0.030$ ); other than skin samples (average integrity $81.2 \% \pm 8.7 \% \mathrm{SE}$ ) vs. indeterminate samples (average integrity $58.5 \%$ $\pm 6.7 \% \mathrm{SE}$ ) (Wilcoxon test corrected for clustered data, adjusted for multiplicity, $\mathrm{p}=0.040$ ). In both cases, significance was confirmed by multivariate quantile regression analysis.

\subsection{DNA mixtures in PHF samples}

PHF samples showing at least one foreign allele were 56 out of 120 (46.7\%). Of these 36 (64.3\%) could be classified as mixtures with an average of 14.5 foreign alleles $(9.2 \pm \mathrm{SD})$, while low-level mixtures were $20(35.7 \%)$. The minimum number of DNA contributors (donor included) was two in $73.2 \%$ of the samples, three in $23.2 \%$ of the samples, and four in $3.6 \%$ of the samples. Among mixed profiles (including low-level mixtures), 18 (32.1\%) displayed all the donors' reference alleles (i.e. $100 \%$ integrity).

Prevalence of mixtures (including low-level mixtures) in PHF samples is summarized in Table 3. The prevalence of mixtures in female PHF samples was 63.3\% (CI 95\% 51.7\%-75.5\%). This was significantly higher if compared to what observed in male PHF samples, where the prevalence was 30\% (CI 95\% 20.0\%- 42.2\%) (Chi-square adjusted for clustered data, $\mathrm{p}=0.005)$. Conversely, 
no significant differences were observed between sampling methods, tissue types and dominant/non-dominant hand.

The distribution of foreign DNA percentages in PHF samples is shown in Figure 2. On average, the percentage of foreign DNA contribution was $8.5 \%( \pm 1.2 \% \mathrm{SE})$. Even though this contribution was $<30 \%$ in most samples, higher proportions of non-self DNA were detected in 11 samples (including 1 low-level mixtures), with a single PHF sample showing a ratio between nonself and self DNA above 1:1 (56.8\%). Foreign DNA contribution was, on average, significantly higher in females $(12.1 \% \pm 1.9 \% \mathrm{SE})$ than in males $(4.9 \% \pm 1.4 \% \mathrm{SE})$ (Wilcoxon test corrected for clustered data, $\mathrm{p}<0.001)$. Significance was confirmed by multivariate quantile regression analysis. It should be noted that, although the percentages mentioned above were obtained by simply measuring the peak heights of expected and unexpected alleles, thus representing an underestimation of foreign DNA (as a matter of fact, we disregarded the possible non-self contribution to imbalanced alleles matching the reference profile), the proportion of mixed profiles showing a foreign contribution $\geq 20 \%$ within the whole palmar sample set $(n=120)$ was $19.2 \%$ (30.0\% in female PHF samples and $8.3 \%$ in male PHF samples) Finally, an inverse correlation between the percentage of foreign DNA contribution and DNA integrity was observed in the same PHF samples (Pearson's coefficient $-0.275, \mathrm{p}=0.001$ ).

\subsection{LCA}

Figure 3 (DNA yield) and Figure 4 (percentage of foreign DNA in mixtures) show the results of the LCA performed on the manifest variables derived from the questionnaire (which is summarized in the supplementary material, Table S2). In the first case, the LCA identified two significantly different profile classes, as class "1" had 14.5 times lower DNA yield than class "2" $(p=0.039)$. The two classes accounted for $44.4 \%$ and $55.6 \%$ of the whole sample set 
respectively. The formation of the two classes was strongly influenced by gender, with $84 \%$ of males in class "2", and 95\% of females in class "1". No other clear dichotomies in the distribution of variables (age, personal and hygiene habits) were observed, with the exception of touching and playing with hair, as this phenomenon was strongly linked to class " 1 " with a probability of $96 \%$. With regard to the percentage of foreign DNA in mixtures, the LCA identified three profile classes with distinct but not significantly different trends: subjects in class "1", which accounted for $29.6 \%$ of the whole sample set, had foreign DNA percentage values three times higher than those in class " 2 " $(p=0.222)$, and two times higher than those in class " 3 " $(p=0.440)$, which represented $41.7 \%$ and $28.7 \%$ of the whole sample set respectively. With regard to the extreme classes "1" and "2", the manifest variables with the most skewed distribution patterns were: female gender (100\% probability in class " 1 "), use of public transport (78.5\% probability in class "1") and living with parents (94.9\% probability in class “2").

\section{Discussion}

mRNA profiling results indicated a relatively high prevalence (15\%) of body fluids different from skin on the palmar surface of the hands and fingers of the tested individuals. Blood and saliva were the most commonly observed body fluids, whereas the sporadic observation of vaginal mucosa may be partly the consequence of MUC4 and HBD1 expression in other mucous membranes, like oral or nasal mucosa, found on human hands [28,29]. PHF samples classified as other than skin showed significantly higher DNA yield and integrity, thus supporting the hypothesis that transfer of cellular material different from skin can be a possible explanation for the occasional recovery of quality STR profiles from handled items.

A consequence of the presence of other than skin cellular material on the palmar surface of the hand and fingers is that, similarly to secondary DNA transfer, the issue of the secondary transfer 
of biological material (i.e., the indirect deposition on a surface of a body fluid carried by the hands of an intermediary who may, or may not, be the primary source of the same) should be taken into account in criminal investigations, especially when dealing with trace amounts of biological evidence. Secondary transfer is a complex phenomenon, which is significantly affected by: the nature of primary and secondary substrates; the type of contact between surfaces; the dryness/wetness of the biological sample [30,31]. Many of these points could not be properly addressed in the present study, since the choice of secondary substrates (dry and wet swabs, stubs) and the type of contact applied (rubbing/tape-lifting of the whole palmar surface of the hand and fingers) were clearly not meant to reproduce real transfer scenarios. However, some interesting considerations can be made. It has been previously shown that no difference in relation to transferability is found between body fluids such as blood and saliva, and that wet samples are much more likely to be transferred than dry samples [30]. On the contrary, similar transfer rates have been described for both freshly deposited and dried skin cells [32]. To explain this difference it has been suggested that skin cells are present within a composite including sweat and oil which may favor the transfer of biological material [32]. However, the hydrolipidic film found on the skin surface may also positively affect the transfer rate of dry deposits of other than skin material. Consequently, a similar propensity in terms of transfer through handling can be hypothesized for both skin cells and other than skin material found on the palmar surface of the hand and fingers. In this respect, it is worth noting that the vast majority of the mRNA profiles from the other than skin palmar samples analyzed in the present study also included skin markers. Thus, the simultaneous detection of skin and other body fluids on an object/surface, when combined with the results of DNA profiling, can aid to discriminate between secondary and primary transfer. 
Another interesting observation emerging from the mRNA profiling study was related to the poor results of the RNA analysis of the samples collected by means of wet swabs. In forensic investigations, the use of a wet cotton swab followed by a second dry swab, the so-called "double swab" technique, is a common method for collecting cellular material from objects [33]. In our experiments, wet swabs appeared to be highly efficient tools to collect DNA. Nevertheless, it is well established that whereas DNA is inherently resistant to spontaneous hydrolysis, RNA is susceptible to hydrolytic breakdown even in aqueous pH-neutral solutions [34]. Non-enzymatic hydrolysis of RNA may therefore explain the higher percentage of indeterminate mRNA profiling results obtained from wet swabs if compared to the ones obtained from dry swabs and stubs. Tape-lifting by means of stubs and the double swab method have been shown to perform equally well in securing epithelial cells from different surfaces, including skin [35]. However, further research is required to investigate whether tape-lifting could represent a preferable sampling technique for stains that must undergo DNA/RNA co-analysis, and to test the efficiency of moistening media different from water, such as RNA stabilizing solutions and ethanol. A serious limitation of the present study was the impossibility to simultaneously apply different sampling methods, while collecting PHF samples from a single donor, due to the unknown spatial distribution of cellular material on the sampled surfaces, thus preventing direct comparison. Moreover, the fact that an individual cannot be relied upon to carry on his hands a consistent amount of cellular material over time [13,32] advised against testing the same donor repeatedly, using different sampling techniques, so as to obtain replicates. The use of known volumes of easily manipulated biological fluids (such as blood or saliva), rather than PHF samples, appears therefore necessary to create the controlled test conditions required for future studies of RNA integrity in stains collected with different sampling methods. 
Apart from tissue origin, the other relevant factor influencing DNA yield and integrity in PHF samples was gender, with males showing higher DNA yield and integrity of the STR profiles than females. This is in contrast with the results of the study where Lowe et al. [12] introduced the "shedder status" and stated that no gender bias could be observed in shedder type. It should be noted, however, that their experiments were based on a limited number of samples (i.e., eight individuals) [12]. In a later study challenging the concept of "poor" and "good" DNA shedder, Phipps et al. [13] completely omitted to consider gender as a potentially important variable. Since then, the relationship between gender and the propensity to transfer DNA has remained poorly investigated [2,3]. In our study and, more specifically, on the basis of the answers to the questionnaire (data not shown), no significant differences between males and females were observed with respect to hygiene (last hand-washing event) and personal (putting hands and objects in the mouth, putting hands in the hair) habits. However, several previous reports in the literature agree that female young adults generally have a better hand hygiene [36-39], possibly due to a higher and more widespread tendency to practice socially acceptable behaviors; this fact is also reflected in our results, especially when the considerably wider range of biological fluids detected on male palmar surfaces is observed. In males, lower hand hygiene compliance and a consequent accumulation of DNA seem therefore to be the most likely explanations for gender bias in DNA quantity and quality. No variables other than gender could be clearly identified by the LCA as being associated with individuals who had a stronger probability to carry high amounts of DNA on their hands, with the partial exception of the absence of a tendency to touch or to play with hair. In particular, no clear subdivision was found in relation to age, which has recently been described as a factor that may possibly influence the extent of DNA transfer [40]. Nevertheless, it is worth noting that in the aforementioned study by Poetsch et al. [40], a 
significant effect of age was exclusively observed for children (good shedders) and elderly people (poor shedders), whereas the present study tested volunteers who were mostly young adults.

STR profiling allowed us to estimate a prevalence of mixtures in PHF samples (disregarding lowlevel mixtures) of $30.0 \%$ (36 samples out of 120). In particular, samples showing a foreign DNA contribution $\geq 20 \%$ (which therefore required a mixture analysis also for the assessment of the genotype of the major contributor, in conformity with current guidelines [41]) were 19.2\% (23 samples out of 120). Bearing in mind the possible differences in the criteria adopted to define DNA mixtures, the prevalence observed in this study is consistent with previous studies of fingernail samples collected from the general population, where values ranged between $13 \%$ and $41 \%$ [17-19]. The analysis of fingernail specimens collected from a victim to identify the DNA traces of his/her assailant is now routine in the forensic investigation of violent crimes and of intimate partner violence $[42,43]$. Our results suggest that sampling the victim's palmar surface of the hands and fingers at post-mortem examination may also be a useful method for the identification of the persons who had contact with the victim prior to death, especially when no signs of a violent struggle are found. Nevertheless, it is to be expected that, even more than in the case of fingernail samples $[43,44]$, palmar DNA mixtures may derive from innocent contact, especially between intimate partners. LCA did not demonstrate any clear association between types of physical contact in the last 24 hours and foreign DNA percentages in PHF samples, possibly due the limited array of activities observed in the donor population (with most participants to the study reporting no other type of physical contact but handshake). LCA suggested a possible influence of house sharing on non-self DNA levels in PHF samples. Nevertheless, no samples were collected from partners, housemates, etc. to generate the related reference STR profiles. So, further studies performed in controlled settings and focusing on a wide range of physical interactions (including sexual intercourse) occurring within a short time 
frame prior to sampling are required to better understand the evidential value of mixed PHF samples in subjects living with other people.

A final interesting observation was that the prevalence of DNA mixtures as well as the percentage of foreign DNA contribution were significantly higher in female rather than in male donors. These results are diametrically opposed to what previously described for fingernail samples, as both Cook et al. [17] and Dowlman et al. [18] reported a higher prevalence of mixtures in male volunteers from the general population. It can be postulated that the lower amount and integrity of self DNA found, on average, on the hands and fingers of female donors favored the amplification of foreign DNA, which is easily transferred to hands through social interaction and object handling. In males, instead, more lax hygiene habits leading to less frequent and less active cleaning of subungual spaces (where penetration of foreign materials is generally related to physical contacts that are more intimate than simple social interactions and object handling) may explain the higher prevalence of DNA mixtures reported in the literature $[17,18]$.

\section{Concluding remarks}

The present research was designed as a cross-sectional study aimed at exploring the touch DNA phenomenon in the general population and in everyday activities. In this respect, it differs from previous works, which were often carried out in artificial settings (e.g., asking volunteers to wash their hands and then perform, or not perform, specific actions). Strict control of experimental conditions is obviously required to reduce the number of variables at play, but it involves the risk of underestimating the complexity of the above-mentioned phenomenon, thus leading to rather simplistic conclusions. Our data confirm, for example, that the definition of "shedder status" is far from easy to assess, with variables such as gender that have been poorly investigated and may play a greater role than what previously estimated. Further work, including an analysis of larger 
and more diverse experimental samples as well as a study of the DNA/RNA transfer and persistence after different types of contact, is therefore necessary to better support "activity level" inferences, which will enable to create a link between touch DNA recovery and crime dynamics in the future.

\section{Acknowledgments}

The continuing support of Compagnia di San Paolo and Fondazione CRT to C. Robino's laboratory is gratefully acknowledged.

\section{References}

1. R.A.H. van Oorschot, M.K. Jones, DNA fingerprints from fingerprints, Nature 387 (1997) 767.

2. R.A.H. van Oorschot, K.N. Ballantyne, R.J. Mitchell, Forensic trace DNA: a review, Investig. Genet. 1 (2010) 14.

3. G. Meakin. A. Jamieson, DNA transfer: Review and implications for casework, Forensic Sci. Int. Genet. 7 (2013) 434-443.

4. F. Alessandrini, M. Cecati, M. Pesaresi, C. Turchi, F. Carle, A. Tagliabracci, Fingerprints as evidence for a genetic profile: morphological study on fingerprints and analysis of exogenous and individual factors affecting DNA typing, J. Forensic Sci. 48 (2003) 586592.

5. T. Kita, H. Yamaguchi, M. Yokoyama, T. Tanaka, N. Tanaka, Morphological study of fragmented DNA on touched objects, Forensic Sci. Int. Genet. 3 (2008) 32-36.

6. I. Quinones, B. Daniel, Cell free DNA as a component of forensic evidence recovered from touched surfaces, Forensic Sci. Int. Genet. 6 (2012) 26-30. 
7. S. Zoppis, B. Muciaccia, A. D'Alessio, E. Ziparo, C. Vecchiotti, A. Filippini, DNA fingerprinting secondary transfer from different skin areas: Morphological and genetic studies, Forensic Sci. Int. Genet. 11 (2014) 137-143.

8. K.A. Reynolds, P. M. Watt, S. A. Boone, C. P. Gerba, Occurrence of bacteria and biochemical markers on public surfaces, Int. J. Environ. Health Res. 15 (2005) 225-234.

9. N.J. Port, V.L. Bowyer, E.A. Graham, M.S. Batuwangala, G.N. Rutty, How long does it take a static speaking individual to contaminate the immediate environment?, Forensic Sci. Med. Pathol. 2 (2006) 157-163.

10. G.N. Rutty, A. Hopwood, V. Tucker, The effectiveness of protective clothing in the reduction of potential DNA contamination of the scene of crime, Int. J. Legal Med. 117 (2003) $170-174$

11. D.H. Warshauer, P. Marshall, S. Kelley, J. King, B. Budowle, An evaluation of the transfer of saliva-derived DNA, Int. J. Legal Med. 126 (2012) 851-861.

12. A. Lowe, C. Murray, J. Whitaker, G. Tully, P. Gill, The propensity of individuals to deposit DNA and secondary transfer of low level DNA from individuals to inert surfaces, Forensic Sci. Int. 129 (2002) 25-34.

13. M. Phipps, S. Petricevic, The tendency of individuals to transfer DNA to handled items, Forensic Sci. Int., 168 (2007) 162-168.

14. V. Castella, P. Mangin, DNA profiling success and relevance of 1739 contact stains from caseworks, Forensic Sci. Int. Genet. Suppl. Ser. 1 (2008) 405-407.

15. J. Sewell, I. Quinones, C. Ames, B. Multaney, S. Curtis, et al., Recovery of DNA and fingerprints from touched documents, Forensic Sci. Int. Genet. 2 (2008) 281-285. 
16. J.J. Raymond, R.A.H. van Oorschot, P.R. Gunn, S. Walsh, C. Roux, Trace evidence characteristics of DNA: a preliminary investigation of the persistence of DNA at crime scenes, Forensic Sci. Int. Genet. 4 (2009) 26-33.

17. O. Cook, Dixon L, The prevalence of mixed DNA profiles in fingernail samples taken from individuals in the general population, Forensic Sci. Int. Genet. 1 (2007) 62-68.

18. E.A. Dowlman, N. C. Martin, M. J. Foy, T. Lochner, T. Neocleous, The prevalence of mixed DNA profiles on fingernail swabs, Sci. Justice 50 (2010) 64-71.

19. M. Matte, L. Williams, R. Frappier, J. Newman, Prevalence and persistence of foreign DNA beneath Fingernails, Forensic Sci. Int. Genet. 6 (2012) 236-243.

20. C. Haas, E. Hanson, J. Ballantyne, Capillary electrophoresis of a multiplex reverse transcription-polymerase chain reaction to target messenger RNA markers for body fluid identification, Methods Mol. Biol, 830 (2012) 169-83.

21. M. Visser, D. Zubakov, K.N. Ballantyne, M. Kayser. mRNA-based skin identification for forensic applications, Int. J. Legal Med. 125 (2011) 253-63.

22. E. Hanson, C. Haas, R. Jucker, J. Ballantyne. Specific and sensitive mRNA biomarkers for the identification of skin in 'touch DNA' evidence, Forensic Sci. Int. Genet. 6 (2012):54858.

23. A. Lindenbergh, M. Pagter de, G. Ramdayal, M. Visser, D. Zubakov, et al., A multiplex (m)RNA-profiling system for the forensic identification of body fluids and contact traces, Forensic Sci. Int. Genet. 6 (2012) 565-577.

24. A. Lindenbergh, P. Maaskant, T. Sijen, Implementation of RNA profiling in forensic casework, Forensic Sci Int Genet 7 (2013) 159-166.

25. A. Donner, Statistical methods in ophthalmology: an adjusted chi-square approach, Biometrics 45 (1989) 695-611. 
26. S. Datta, G.A. Satten, Rank-sum tests for clustered data, J. Am. Stat. Assoc., 100 (2005) 908-915.

27. R Core Team. R: A Language and Environment for Statistical Computing (2015) R Foundation for Statistical Computing, Vienna, Austria.

28. Y. Xu, J. Xie, Y. Cao, H. Zhou, Y. Ping, L. Chen, L. Gu, W. Hu, G. Bi, J. Ge, Development of highly sensitive and specific mRNA multiplex system (XCYR1) for forensic human body fluids and tissues identification, PLoS One 9 (2014) e100123.

29. C. Cossu, U. Germann, A. Kratzer, W. Baer, C. Haas, How specific are the vaginal secretion mRNA-markers HBD1 and MUC4, Forensic Sci. Int. Genet. Suppl. Ser. 2 (2009) 536-537.

30. M. Goray, E. Eken, R.J. Mitchell, R.A.H. van Oorschot, Secondary DNA transfer of biological substances under varying test conditions, Forensic Sci. Int. Genet. 4 (2010) 6267.

31. T.J. Verdon, R.J. Mitchell, R.A.H. van Oorschot, The influence of substrate on DNA transfer and extraction efficiency, Forensic Sci. Int. Genet. 7 (2013) 167-175.

32. M. Goray, R.J. Mitchell, R.A.H. van Oorschot, Investigation of secondary DNA transfer of skin cells under controlled conditions, Leg. Med. (Tokyo) 12 (2010) 117-120.

33. B.C.M. Pang, B.K.K. Cheung, Double swab technique for collecting touched evidence, Leg. Med. (Tokyo) 9 (2007) 181-184.

34. F.H. Westheimer, Why nature chose phosphates, Science 235 (1987) 1173-1178.

35. K.G. de Bruin, S.M. Verheij, M. Veenhoven, T. Sijen. Comparison of stubbing and the double swab method for collecting offender epithelial material from a victim's skin, Forensic Sci. Int. Genet. 6 (2012) 219-223. 
36. D. Drankiewicz, L. Dundes, Handwashing among female college students, Am. J. Infect. Control 31 (2003) 67-71

37. H.D. Johnson, D. Sholcosky, K. Gabello, R. Ragni, N. Ogonosky, Sex differences in public restroom handwashing behavior associated with visual behavior prompts, Percept. Mot. Skills 97 (2003) 805-810.

38. C. White, R. Kolble, R. Carlson, N. Lipson, The impact of a health campaign on hand hygiene and upper respiratory illness among college students living in residence halls, J. Am. Coll. Health 53 (2005) 175-181

39. J. L. Anderson, C.A. Warren, E. Perez, R.I. Louis, S. Phillips, et al., Gender and ethnic differences in hand hygiene practices among college students, Am. J. Infect. Control 36 (2008) $361-368$

40. M. Poetsch, T. Bajanowski and T. Kamphausen, Influence of an individual's age on the amount and interpretability of DNA left on touched items, Int J. Legal Med. 127 (2013) 1093-1096.

41. P.M. Schneider, R. Fimmers, W. Keil, G. Molsberger, D. Patzelt et al. The German Stain Commission: recommendations for the interpretation of mixed stains, Int J. Legal Med. $123(2009) 1-5$.

42. B. Nurit, G. Anat, S. Michal, F. Lilach, F. Maya, Evaluating the prevalence of DNA mixtures found in fingernail samples from victims and suspects in homicide cases, Forensic Sci. Int. Genet. 5 (2011) 532-527.

43. M. Kettner, S. Cappel-Hoffmann, D. Makuch, P. Schmidt, F. Ramsthaler. IPV--bridging the juridical gap between scratches and DNA detection under fingernails of cohabitating partners, Forensic Sci. Int. Genet. 14 (2015) 110-115. 
44. S. Malsom, N. Flanagan, C. McAlister, L. Dixon, The prevalence of mixed DNA profiles in fingernail samples taken from couples who co-habit using autosomal and Y-STRs, Forensic Sci. Int. Genet. 2 (2009) 57-62. 
Table 1

\begin{tabular}{|c|c|c|c|c|c|c|c|}
\hline \multirow{3}{*}{$\begin{array}{c}\text { mRNA } \\
\text { profiling }\end{array}$} & \multicolumn{3}{|c|}{ Sampling Method } & \multicolumn{2}{c|}{ Gender } & \multicolumn{2}{c|}{ Hand dominance } \\
\cline { 2 - 8 } & $\begin{array}{c}\text { Dry } \\
\text { swab } \\
(n=40)\end{array}$ & $\begin{array}{c}\text { Wet } \\
\text { swab } \\
(n=40)\end{array}$ & $\begin{array}{c}\text { Stub } \\
(n=40)\end{array}$ & $\begin{array}{c}\text { Male } \\
(n=60)\end{array}$ & $\begin{array}{c}\text { Female } \\
(n=60)\end{array}$ & $\begin{array}{c}\text { Dominant } \\
(n=60)\end{array}$ & $\begin{array}{c}\text { Non } \\
\text { dominant } \\
(n=60)\end{array}$ \\
\hline Skin & $\begin{array}{c}25 \\
(63 \%)\end{array}$ & $\begin{array}{c}20 \\
(50 \%)\end{array}$ & $\begin{array}{c}23 \\
(58 \%)\end{array}$ & $\begin{array}{c}31 \\
(52 \%)\end{array}$ & $\begin{array}{c}37 \\
(62 \%)\end{array}$ & $\begin{array}{c}36 \\
(60 \%)\end{array}$ & $\begin{array}{c}32 \\
(53 \%)\end{array}$ \\
\hline Other than skin & 9 & 2 & 7 & 13 & 5 & 9 \\
$(8 \%)$ & $(18 \%)$ & $\begin{array}{c}9 \\
(15 \%)\end{array}$ \\
\hline Indeterminate & 6 & 18 & 10 & 16 & 18 & 15 & 19 \\
& $(15 \%)$ & $(45 \%)$ & $(25 \%)$ & $(27 \%)$ & $(30 \%)$ & $(25 \%)$ & $(32 \%)$ \\
\hline
\end{tabular}

Table 2

\begin{tabular}{|l|c|c|c|}
\hline & $\begin{array}{c}\text { Average DNA } \\
\text { integrity } \%\end{array}$ & \multicolumn{2}{|c|}{ Comparisons (Wilcoxon test) } \\
\hline Sampling Method & & Dry swab vs. Wet swab & $\mathrm{ns}$ \\
\hline Dry swab $(n=40)$ & $60.3 \%( \pm 6.8 \%)$ & Dry swab vs. Stub & $\mathrm{ns}$ \\
\hline Wet swab $(n=40)$ & $70.9 \%( \pm 5.2 \%)$ & Met swab vs. Stub & \\
\hline Stub $(n=40)$ & $71.8 \%( \pm 6.9 \%)$ & & $\mathrm{p}=0.030$ \\
\hline Gender & & & $\mathrm{ns}$ \\
\hline Male $(n=60)$ & $60.1 \%( \pm 5.2 \%)$ & Dominant vs. Non dominant & \\
\hline Female $(n=60)$ & & & $\mathrm{ns}$ \\
\hline Hand dominance & $69.8 \%( \pm 5.1 \%)$ & Skin vs. Other than skin & \\
\hline Dominant $(n=60)$ & $65.4 \%( \pm 5.3 \%)$ & & \\
\hline Non dominant $(n=60)$ & $68.6 \%( \pm 5.0 \%)$ & & \\
\hline Tissue type & $81.2 \%( \pm 8.7 \%)$ & & \\
\hline Skin $(n=68)$ & & & \\
\hline Other than skin $(n=18)$ & & & \\
\hline
\end{tabular}


Table 3

\begin{tabular}{|c|c|c|c|}
\hline \multirow[b]{2}{*}{ Sampling Method } & \multirow{2}{*}{$\begin{array}{l}\text { Prevalence } \\
\text { of mixtures }\end{array}$} & \multicolumn{2}{|c|}{ Comparisons (Chi-square test) } \\
\hline & & & \\
\hline Dry swab $(n=40)$ & $\begin{array}{c}47.5 \% \\
(\mathrm{CI} 95 \% 32.5 \%-62.5 \%)\end{array}$ & Dry swab vs. Wet swab & ns \\
\hline Wet swab $(n=40)$ & $\begin{array}{c}40.0 \% \\
(\mathrm{CI} 95 \% 27.5 \%-56.9 \%)\end{array}$ & Dry swab vs. Stub & ns \\
\hline $\operatorname{Stub}(n=40)$ & $\begin{array}{c}52.5 \% \\
(\text { CI } 95 \% 37.5 \%-67.5 \%)\end{array}$ & Wet swab vs. Stub & ns \\
\hline Gender & & & \\
\hline Male $(n=60)$ & $\begin{array}{c}30.0 \% \\
(\text { CI } 95 \% 20.0 \%-42.4 \%)\end{array}$ & Male vs. Female & $\mathrm{p}=0.005$ \\
\hline Female $(n=60)$ & $\begin{array}{c}63.3 \% \\
(\mathrm{CI} 95 \% 51.7 \%-75.5 \%)\end{array}$ & & \\
\hline Hand dominance & & & \\
\hline Dominant $(n=60)$ & $\begin{array}{c}48.3 \% \\
(\mathrm{CI} 95 \% 36.7 \%-61.8 \%)\end{array}$ & Dominant vs. Non dominant & ns \\
\hline Non dominant $(n=60)$ & $\begin{array}{c}45.0 \% \\
(\mathrm{CI} 95 \% 33.3 \%-58.3 \%)\end{array}$ & & \\
\hline Tissue type & & & \\
\hline $\operatorname{Skin}(n=68)$ & $\begin{array}{c}42.6 \% \\
(\text { CI 95\% 32.3\% - 55.6\%) }\end{array}$ & Skin vs. Other than skin & ns \\
\hline
\end{tabular}




\begin{tabular}{|l|c|c|c|}
\hline Other than skin $(n=18)$ & $33.3 \%$ & Skin vs. Indeterminate & ns \\
\hline Indeterminate $(n=34)$ & $($ CI $95 \% 16.7 \%-56.9 \%)$ & & ns \\
\hline
\end{tabular}

\section{Table 1}

Distribution of tissue type categories in PHF samples subdivided by: a) sampling method; b) gender; c) hand dominance. Absolute frequencies are shown (percentages are given between brackets).

\section{Table 2}

Average DNA integrity percentages in PHF samples subdivided by: a) sampling method; b) gender; c) hand dominance; d) tissue type category. Standard error is given between brackets. Pvalues of Wilcoxon test are shown in the column to the right (ns denotes non-significance).

\section{Table 3}

Prevalence of mixtures (including low-level mixtures) in PHF samples subdivided by: a) sampling method; b) gender; c) hand dominance; d) tissue type category. 95\% confidence intervals are given between brackets. P-values of Chi-square test are shown in the column to the right (ns denotes non-significance).

\section{Figure 1}


Box-and-whisker plots describing the log scale distribution of the amount of DNA (ng) recovered from PHF samples ( $n=120)$ subdivided by: a) sampling method; b) gender; c) hand dominance; d) tissue type category.

\section{Figure 2}

Distribution of the percentages of foreign DNA contribution in PHF samples $(n=120)$. Mixtures are shown in dark color, low-level mixtures in medium color, samples with no evidence of foreign alleles in light color.

\section{Figure 3}

Output of the LCA performed to identify the profiles associated with different DNA yields: class "1", continuous line; class "2", dashed line. Composition of profile classes associated with low ("1") and high ("2") DNA yields in PHF samples is shown below. Numbers along the right axis (1-5) indicate category variables, as identified in the first row of Supplementary Table S2. Bar heights represent the probability that a specific category variable is present in class " 1 " or " 2 ".

\section{Figure 4}

Output of the LCA performed to identify the profiles associated with different levels of foreign DNA contribution to mixtures: class "1", continuous line; class "2", dashed line; class "3", dotted line. Composition of profile classes associated with high ("1"), low ("2") and medium ("3") percentages of foreign DNA contribution to DNA mixtures found on PHF samples is shown below. Numbers along the right axis (1-7) indicate category variables, as identified in the first row of Supplementary Table S2. Bar heights represent the probability that a specific category variable is present in class "1", "2" or "3". 